\title{
AVALIAÇÃO PRENSAGEM DE MISTURA DE MINÉRIO DE FERRO E CARVÃO EM ESCALA PILOTO*
}

Catia Casagrande ${ }^{1}$ Francisco Macedo ${ }^{2}$ Simonny Guachalla ${ }^{3}$ Guenter Hahn ${ }^{4}$

\section{Resumo}

O processo de pelotização foi desenvolvido com a finalidade de aproveitar as partículas finas e ultrafinas geradas nas operações de exploração e beneficiamento do minério de ferro. Entretanto para atender as usinas de pelotização, faz-se necessário utilizar a moagem para adequar as características de granulometria e superfície específica das misturas de minérios de ferro às especificações da produção de pelotas. Porém os moinhos possuem alto consumo de energia elétrica, elevando o custo operacional do processo de pelotização. Frente ao cenário atual, foi realizado o trabalho para estudar a substituição à moagem (área úmida) por processo de multi prensagens. Além disso, foram misturados os minérios de ferro com carvão, a fim de reduzir também o custo da moagem deste insumo. O objetivo principal foi quebrar as partículas de mistura de minérios de ferro e carvão, e avaliar os resultados de superfície específica e granulometria deste produto comparando com o processo padrão (moagem e prensagem). A prensa de rolos de bancada e as análises físicas e químicas foram realizadas para o desenvolvimento do trabalho. Foi concluída a possibilidade de atingir granulometria e superfície específica através de multi prensagens, atendendo o processo de pelotamento.

Palavras-chave: Minérios de ferro; Carvão; Multi prensagens; Granulometria.

\section{EVALUATION OF IRON ORE AND COAL MIXTURE BY MULTIPLE HPGR GRINDING IN PILOT PLANT}

\section{Abstract}

The pelletizing process was developed in order to take advantage of fine and ultrafine particles generated in the exploration's operation and the iron ore beneficiation process. However to attend the pelletizing plants, it is necessary to use grinding to adjust the particle size and specific surface area characteristics of iron ore mixtures according to the pellet production specifications. But the mills have high energy consumption, increasing the operational cost of the pelletizing process. The work was done to study the replacement of the mill process (wet area) for multiple HPGR grinding. Furthermore, the iron ores were blended with coal in order to also reduce the cost of the grinding of this raw material. The main objective was to break the particle of coal and iron ore mixture, and to evaluate the specific surface and particle size results of this product compared to the standard process results (milling and pressing). The press rolls bench and the physical and chemical analyses were made for the development of the work. In conclusion, the particle size and specific surface area through multiple HPGR grinding can be achieved, attending the pelletizing process.

Keywords: Iron ore; Coal; Multiple HPGR grinding; Size particle.

\footnotetext{
Engenheira Geóloga, Mestre, Engenheira Sênior, Diretoria de Pelotização, Vale S.A., Vitória, ES, Brasil. Engenheiro Metalurgista, Engenheiro Especialista, Diretoria de Pelotização, Vale S.A., Vitória, ES, Brasil. Engenheira Química, Gerente de Garantia da Qualidade, Diretoria de Pelotização, Vale S.A., Vitória, ES, Brasil.

4 Engenheiro Metalurgista, Gerente da Unidade Técnica das Usinas 1 a 4, Diretoria de Pelotização, Vale S.A., Vitória, ES, Brasil.
} 


\section{INTRODUÇÃO}

Com a finalidade de aproveitar as partículas finas e ultrafinas geradas nas operações de extração e beneficiamento do minério de ferro, foi desenvolvido o processo de pelotização. Um grande complexo industrial, constituído por oito usinas de pelotização, está instalado na Ponta de Tubarão, na cidade de Vitória, ES e em Minas Gerais, mais duas usinas, localizadas em Congonhas e Nova Lima. No Brasil o conjunto destas plantas tem capacidade de produção anual de aproximadamente 56 milhões de toneladas de pelotas. No exterior, a Vale possui uma usina de pelotização localizada em Omã.

O processo de pelotização demanda granulometria mais fina e sua alimentação é realizada com mistura de minérios de ferro provenientes das minas, as quais apresentam tamanho natural inferior a $6 \mathrm{~mm}$ e também dos finos gerados na lava, no processamento de concentração, na classificação, no manuseio e transporte do minério. Na concentração do minério de ferro, gera-se pellet feed. O pellet feed é um material mais fino, com excelente liberação, já que apresenta alta concentração de ferro, ou seja, baixo teor de ganga.

A moagem do minério se torna necessária para adequar as características de granulometria e superfície específica das misturas de minérios de ferro às especificações da produção de pelotas. Quanto maior a quebra das partículas de minério de ferro, maior a energia no processo de moagem.

A moagem é uma das etapas que mais consomem energia em uma usina de pelotização. Os moinhos são equipamentos de alto custo e seu correto dimensionamento consiste, principalmente, em determinar a quantidade de energia que os minérios vão demandar para atingir um determinado tamanho [1].

Enquanto que o processo de prensagem apresenta alta eficiência com baixo consumo de energia elétrica comparado com o processo de moagem.

A Vale é pioneira na utilização de prensa de rolos, tendo instalado e operacionalizado as primeiras prensas para minério de ferro no mundo, no início da década de 90 . Estudos e patente foram desenvolvidos pelo corpo técnico ao longo destes 25 anos, possuindo o maior parque de prensagens em suas plantas de pelotização.

Os insumos são muitos importantes para a aglomeração dos minérios de ferro, sendo o carvão o mais caro entre eles. Outros insumos foram considerados no estudo, mas na presente publicação, serão abordados os resultados relativos a mistura de minério de ferro e carvão na forma de antracito.

O carvão passou a ser utilizado nos processos de pelotização, com o objetivo de se obter uma melhor distribuição de temperatura no interior da pelota durante a etapa de queima, possibilitando uma redução do consumo de óleo combustível ou gás natural dos fornos de pelotização [2].

A figura 1 apresenta um gráfico que mostra o efeito da adição de carvão, representado pelo carbono fixo (\%), na produção de pelota de hematita e magnetita. 


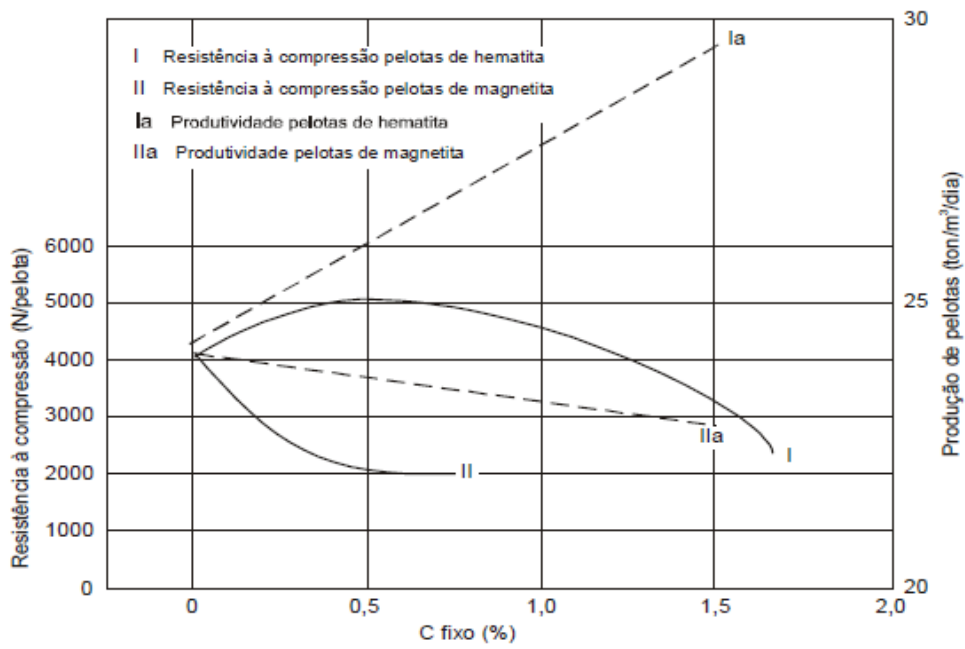

Figura 1. Relação entre a resistência à compressão das pelotas de hematita e magnetita, adição de carvão e produtividade das pelotas durante a queima [3].

Atualmente, no Complexo de Tubarão, realiza-se moagem a úmido de carvão, onde a dosagem é feita antes do processo de mistura, o que reduz a flexibilidade nas alterações no processo. Para trazer redução de custos, ganhos de qualidade e otimização no processo, faz-se necessário avaliar outras formas de quebra das partículas de carvão e outros insumos do processo de pelotização.

Estudos de tecnologia e/ou inovação suportam as possibilidades de modificar o processo, atingindo ganhos nos custos e mesma qualidade.

Ao longo das últimas décadas, a tecnologia de pelotização de minério de ferro tem evoluído consideravelmente [4]. Além de evolução da tecnologia, as empresas visam redução de custo operacional, para adequar ao cenário atual de mineração de ferro.

Frente ao cenário, foi feito um estudo de prensagem com objetivo de cominuir as partículas de minérios de ferro e carvão, assim como avaliar a superfície específica e granulometria deste produto comparando com o processo padrão.

\section{MATERIAIS E MÉTODOS}

A prensa de rolos consiste em dois rolos giratórios opostos acoplados a rolamentos resistentes à tração, revestidos por uma estrutura forte. A pressão é aplicada a um dos rolos por meio de um sistema pneumático de mola, e o outro rolo é preso em uma posição fixa na carcaça.

A cominuição através dos rolos ocorre quando o tamanho máximo da partícula na alimentação é maior que a abertura entre os rolos. A quebra da partícula também ocorre entre partículas.

A prensa de rolos utilizada para o estudo possui um tamanho de $X$, considerada escala de bancada, localizada na Usina Piloto da Diretoria de Pelotização. Para realização dos testes foram fixados parâmetros operacionais, tais como, $20 \mathrm{~kg}$ de alimentação, $8 \%$ de umidade e 80 bar de pressão $\mathrm{N}_{2}$. 


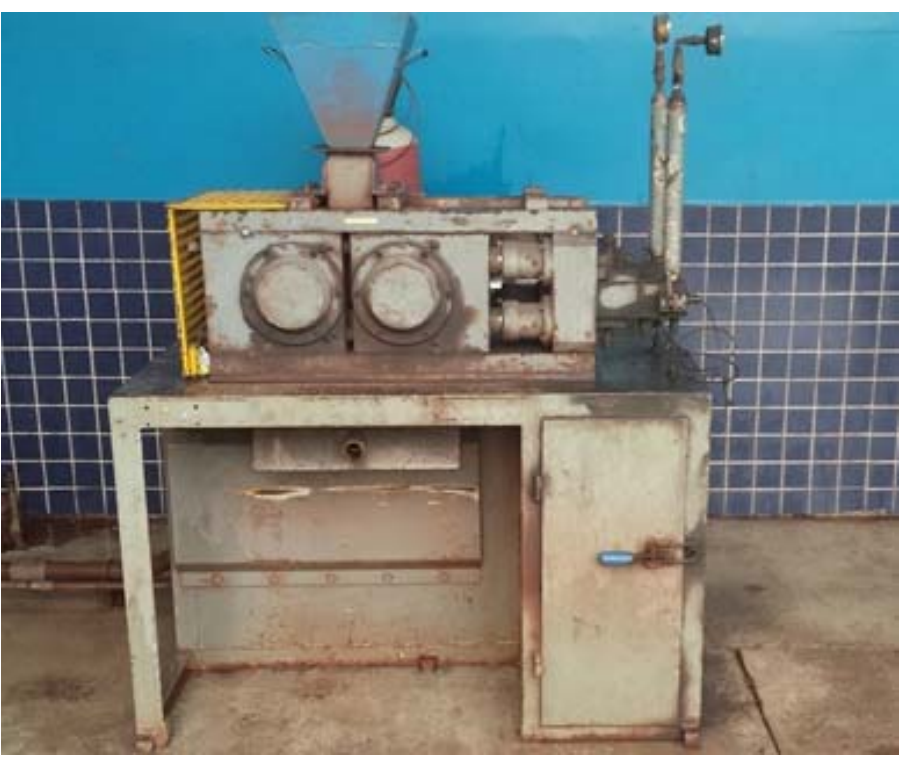

Figura 2. Fotografia da prensa de rolos de bancada.

Os ensaios realizados para avaliar as características físicas do produto foram peneiramento a úmido, fração passante em 45microns pelo método Alpine, e superfície específica através da metodologia Blaine. Normalmente a análise em Alpine apresenta um valor acima do determinado no peneiramento a úmido. Dependendo da avaliação e comparação entre resultados, usa-se uma determinada metodologia.

A granulometria abaixo de 45 microns é controlada no processo de cominuição, sendo moagem ou prensagem. Para realizar um bom pelotamento, o processo exige granulometria acima de $80 \%$ no passante de 45 microns e superfície específica acima de $1800 \mathrm{~cm}^{2} / \mathrm{g}$. Tanto a mistura de minérios de ferro como o carvão foram caracterizados individualmente para avaliar as características químicas e físicas, e posteriormente homogeneizados atendendo a especificação do processo de pelotização. Esta mistura de minérios de ferro e carvão foi processada na prensa de rolos em várias prensagens contínuas, três, quatro e cinco passadas, e caracterizado cada produto destas prensagens.

\section{RESULTADOS E DISCUSSÃO}

A mistura de minérios de ferro foi analisada quimicamente como pode ser visto na Tabela 1

Tabela 1. Resultados de análise química da mistura de minérios de ferro

\begin{tabular}{lc}
\multicolumn{1}{c}{ Parâmetros } & Resultados \\
\hline $\mathrm{Fe}_{\text {cal }}$ & $68,42 \%$ \\
\hline $\mathrm{SiO}_{2}$ & $1,16 \%$ \\
\hline $\mathrm{Al}_{2} \mathrm{O}_{3}$ & $0,29 \%$ \\
\hline $\mathrm{P}$ & $0,014 \%$ \\
\hline $\mathrm{Mn}$ & $0,035 \%$ \\
\hline $\mathrm{CaO}$ & $0,03 \%$ \\
\hline $\mathrm{MgO}$ & $0,01 \%$ \\
\hline $\mathrm{TiO} 2$ & $0,209 \%$ \\
\hline $\mathrm{PPC}$ & 0,40 \\
\hline
\end{tabular}


Em relação à análise química, os resultados apresentaram dentro do projetado. A amostra mistura de minérios de ferro apresentou acima do especificado para pelota de redução direta, porém como foi composta, podem ocorrer estas variações.

A Figura 3 mostra o gráfico dos resultados de granulometria da mistura de minérios de ferro através do peneiramento a úmido.

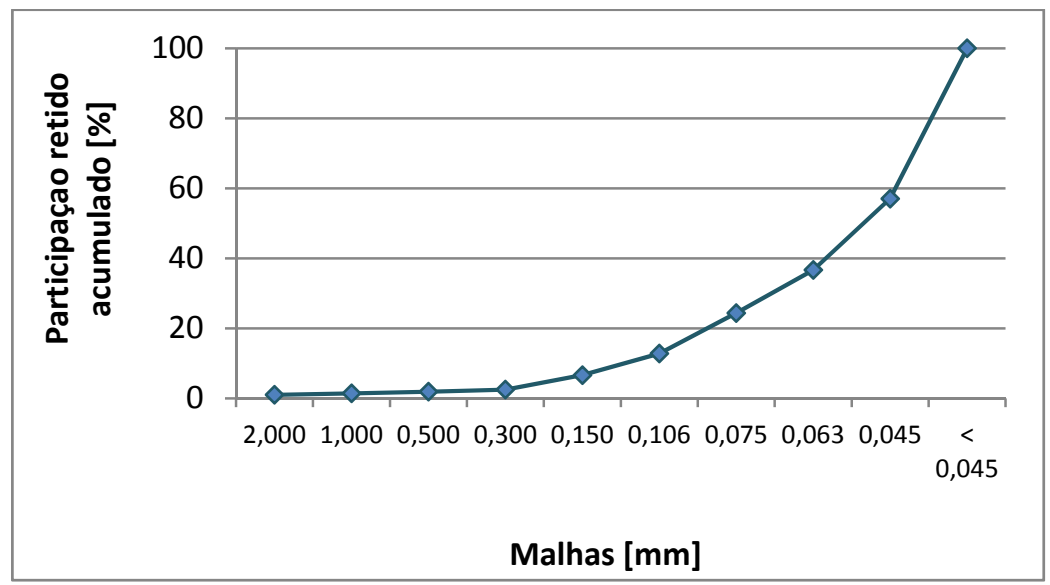

Figura 3. Resultados de granulometria através de peneiramento a úmido da mistura de minérios de ferro.

Como pode ser observada no gráfico da Figura 3, a granulometria do material (\% $<0,045 \mathrm{~mm}$ ), em relação a peneiramento a úmido, é alta, em torno de $43 \%$. Isso influência positivamente no processo de prensagem.

A Tabela 2 mostra a participação abaixo de 45 microns pela metodologia de Alpine e superfície específica da mistura de minérios de ferro. Sabe-se que existe uma diferença entre os resultados de metodologias diferentes, devido à preparação e análise de cada técnica analítica.

Tabela 2. Resultados de granulometria e superfície específica de mistura de minérios de ferro

\begin{tabular}{lc}
\hline Parâmetros & Resultados \\
\hline Superfície específica & $460 \mathrm{~cm}^{2} / \mathrm{g}$ \\
\hline$<0,045 \mathrm{~mm}$ & $48,07 \%$ \\
\hline
\end{tabular}

A superfície específica da mistura de minério de ferro é baixa, enquanto que a granulometria abaixo de $0,045 \mathrm{~mm}$ é alta, quando comparado estes parâmetros com as misturas recebidas nas usinas industriais.

A Tabela 3 apresenta os resultados de análise imediata do carvão.

Tabela 3. Resultados de análise imediata do carvão

\begin{tabular}{lc}
\hline Parâmetros & Resultados \\
\hline Matéria Volátil & $12,34 \%$ \\
\hline Carbono fixo & $68,90 \%$ \\
\hline Cinzas & $18,83 \%$ \\
\hline Umidade & $8,19 \%$ \\
\hline
\end{tabular}

Conforme os resultados de análise imediata nota-se elevados o teor de matéria volátil e cinzas do carvão, porém mesmo com estes valores altos, o mesmo pode ser considerado como um antracito. 
A Figura 4 apresenta a distribuição granulométrica do carvão através do peneiramento a úmido.

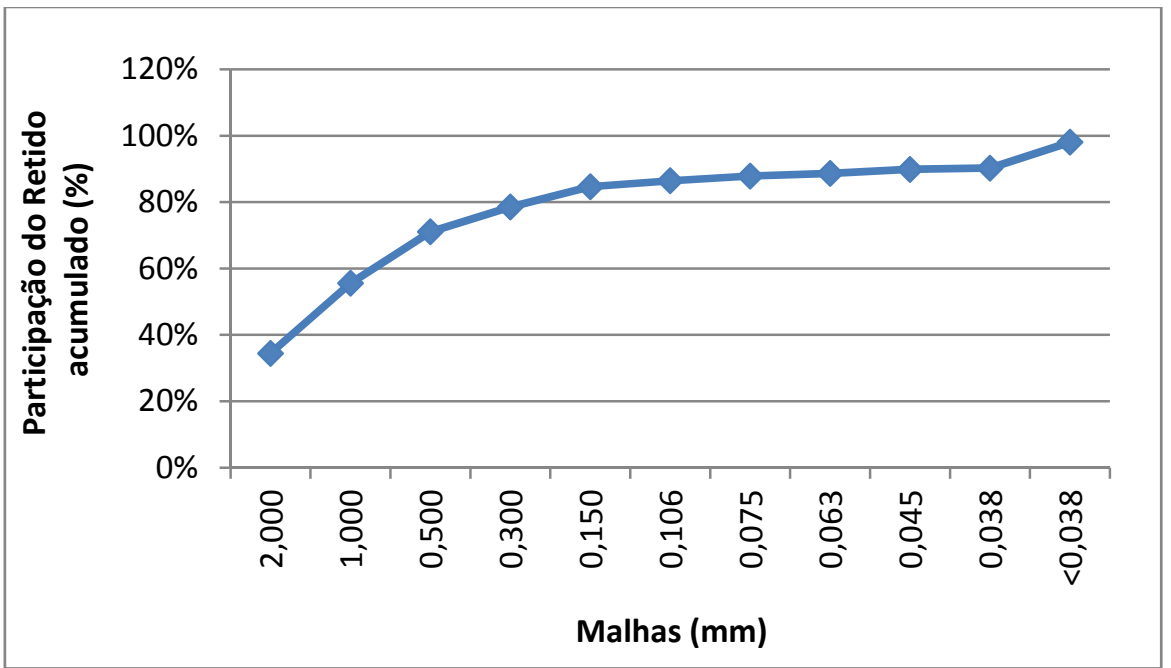

Figura 4. Resultados de granulometria através de peneiramento a úmido do carvão.

Observa-se através dos resultados do gráfico da Figura 3 que o carvão apresenta granulometria grosseira, sendo $84,64 \%$ na fração retida acumulado em $0,150 \mathrm{~mm}$. Entretanto, foi observada também uma participação significativa $(7,80 \%)$ na fração abaixo de 0,038mm.

As cinzas do carvão foram analisadas quimicamente para posteriormente verificar as características químicas da mistura de minérios de ferro e carvão.

Tabela 4. Resultados de análise química das cinzas do carvão

\begin{tabular}{lc}
\hline Parâmetros & Resultados \\
\hline $\mathrm{Fe}_{\text {cal }}$ & $6,80 \%$ \\
\hline $\mathrm{SiO}_{2}$ & $62,11 \%$ \\
\hline $\mathrm{Al}_{2} \mathrm{O}_{3}$ & $19,82 \%$ \\
\hline $\mathrm{M}$ & $0,133 \%$ \\
\hline $\mathrm{Mn}$ & $0,029 \%$ \\
\hline $\mathrm{MgO}$ & $2,94 \%$ \\
\hline $\mathrm{TiO}_{2}$ & $0,88 \%$ \\
\hline $\mathrm{Na}_{2} \mathrm{O}$ & $1,216 \%$ \\
\hline $\mathrm{K}_{2} \mathrm{O}$ & $0,80 \%$ \\
\hline
\end{tabular}

Observa-se que a sílica encontrada nas cinzas do carvão é alta, aproximadamente de $62 \%$. A caracterização química na amostra individual de carvão é importante para avaliação da fração que se encontra o carvão na mistura de minérios de ferro e carvão.

A Tabela 5 mostra os resultados de granulo-química da mistura de minérios de ferro e carvão antes de processar em prensa de rolos. 
Tabela 5. Resultados de análise granulo-química das misturas de minérios de ferro e carvão

\begin{tabular}{cccccc}
\hline Parâmetros & $\mathbf{+ 0 , 1 5 0 m m}$ & $\mathbf{+ 0 , 0 6 3 m m}$ & $\mathbf{+ 0 , 0 4 5 m m}$ & $\mathbf{+ 0 , 0 3 8 m m}$ & $\mathbf{- 0 , 0 3 8} \mathbf{m m}$ \\
\hline $\mathrm{Fe}_{\text {ootal }}(\%)$ & 56,15 & 68,41 & 68,31 & 68,23 & 68,21 \\
\hline $\mathrm{SiO}_{2}(\%)$ & 14,47 & 1,21 & 1,23 & 1,21 & 1,33 \\
\hline $\mathrm{Al}_{2} \mathrm{O}_{3}(\%)$ & 1,16 & 0,33 & 0,21 & 0,24 & 0,24 \\
\hline $\mathrm{P}(\%)$ & 0,039 & 0,016 & 0,011 & 0,013 & 0,013 \\
\hline $\mathrm{Mn}(\%)$ & 0,056 & 0,036 & 0,024 & 0,039 & 0,042 \\
\hline $\mathrm{CaO}(\%)$ & 0,06 & 0,03 & 0,03 & 0,03 & 0,03 \\
\hline $\mathrm{MgO}(\%)$ & 0,07 & 0,02 & 0,01 & 0,02 & 0,01 \\
\hline $\mathrm{TiO}_{2}(\%)$ & 0,01 & 0,065 & 0,037 & 0,059 & 0,056 \\
\hline $\mathrm{PPC}$ & 3,78 & 0,65 & 0,32 & 0,38 & 0,41 \\
\hline
\end{tabular}

Os resultados apresentados na Tabela 5 mostraram valores elevados de teor de contaminantes na malha mais grosseira $(+0,150 \mathrm{~mm})$, como por exemplo, o teor de sílica, alumina e PPC. O valor de PPC é aproximadamente 5,8 vezes mais do que na malha inferior a esta, e a sílica 14 vezes mais. O que pode ser observado que 0 carvão encontra-se retido na malha mais grosseira $(+0,150 \mathrm{~mm})$.

Os teores do ferro e contaminantes são similares nas malhas abaixo de $0,063 \mathrm{~mm}$. A Figura 5 apresenta os resultados de granulometria após as prensagens, sendo comparadas em 3, 4 e 5 passadas pela prensa de rolos.

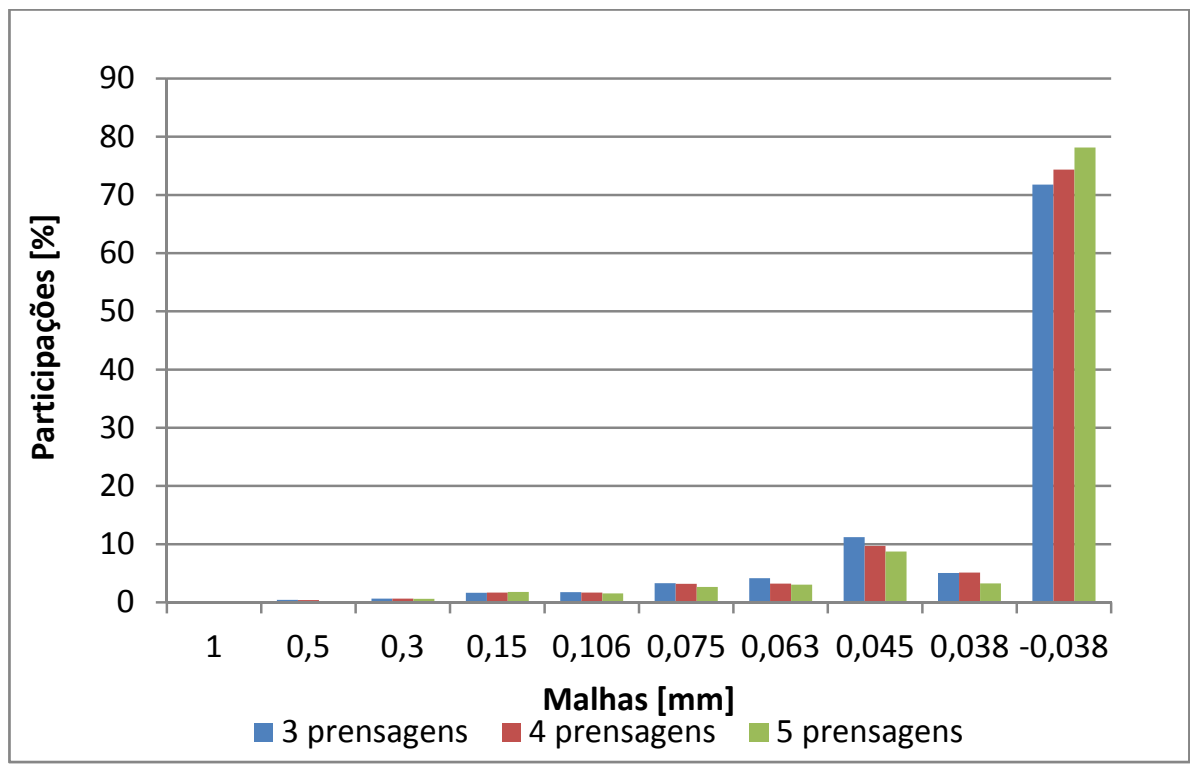

Figura 5. Resultados de granulometria das amostras prensadas.

Observa-se que os resultados de granulometria de cinco passagens em prensa de rolos atende a especificação da planta industrial. Assim como quatro prensagens, na possibilidade de ajustar o processo de pelotamento.

A Figura 6 mostra o gráfico dos resultados de granulometria na malha passante em 0,045mm pelo método Alpine. 


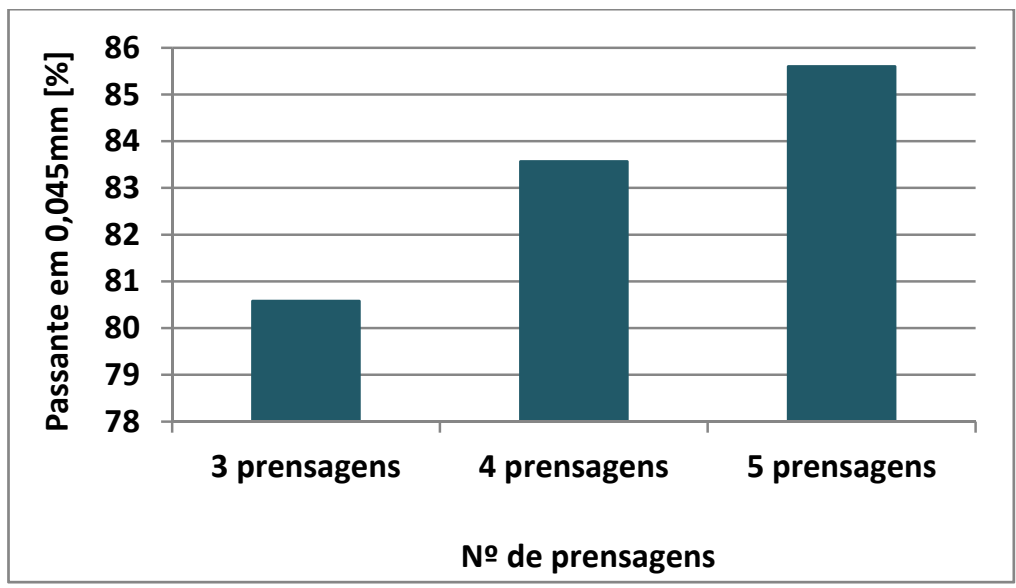

Figura 6. Resultados de granulometria das amostras prensadas.

A granulometria da mistura de minérios de ferro com carvão após 4 passadas em prensa de rolos foi de $83,57 \%$ abaixo de $0,045 \mathrm{~mm}$; enquanto que 5 prensagens foi de $85,60 \%$.

De acordo com os resultados de granulometria por Alpine, o percentual passante em $0,045 \mathrm{~mm}$ acima de 4 prensagens contínuas atende ao processo de pelotamento.

A Figura 7 apresenta os resultados de superfície específica após prensagens.

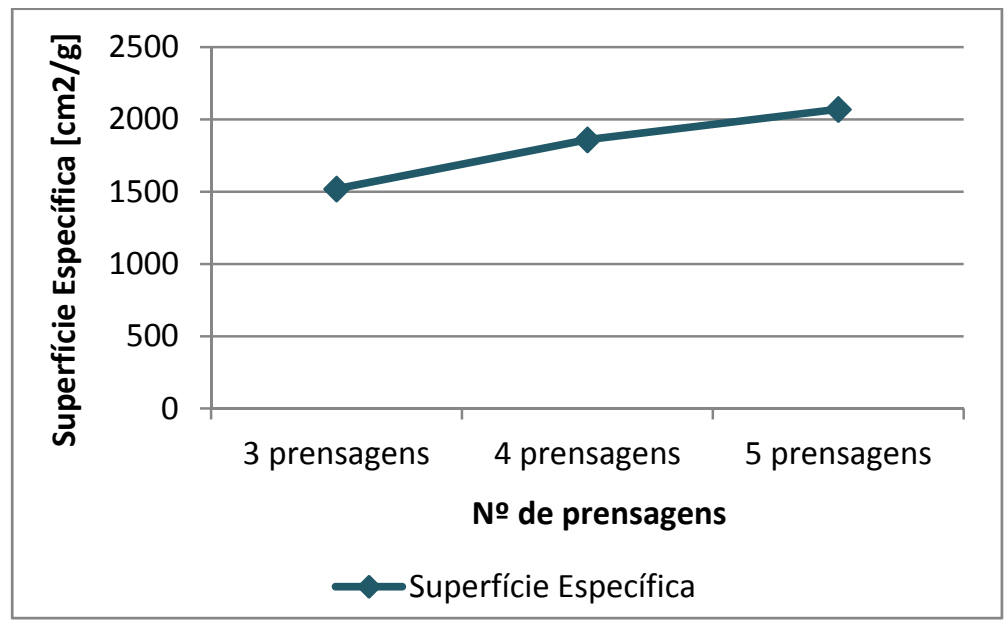

Figura 7. Resultados de superfície específica das amostras prensadas.

A superfície específica da mistura de minérios de ferro com carvão após 4 passadas em prensa de rolos foi de $1860 \mathrm{~cm}^{2} / \mathrm{g}$; enquanto que 5 prensagens foi de $2070 \mathrm{~cm}^{2} / \mathrm{g}$. De acordo com a especificação do processo de pelotização da Vale, para esta mistura de minérios e carvão, acima de quatro prensagens foi atingido o patamar requerido de superfície específica e granulometria para o processo de pelotamento.

\section{CONCLUSÃO}

Devido à granulometria mais grosseira do carvão, comparando com a mistura de minérios de ferro, foi confirmado pelo ensaio de granulo-química que as partículas de carvão ficam retidas na malha mais grosseira (de $0,150 \mathrm{~mm}$ ) muito semelhante a atual condição de aplicação. 
A mistura de minérios de ferro e carvão pode ser submetida a várias prensagens, com possibilidade de otimizar o processo de preparação.

O número de passadas em prensa de rolos para atingir a granulometria (passante em $0,045 \mathrm{~mm}$ ) e superfície específica, para esta mistura avaliada, deve ser acima de quatro, atendendo ao processo de pelotamento.

\section{Agradecimentos}

A Vale por ter nos apoiado e suportado os recursos para realização do estudo. $E$ principalmente aos colegas da Usina Piloto da Diretoria de Pelotização.

\section{REFERÊNCIAS}

1 Ribeiro MR. Investigação das características dos minérios de ferro da CVRD que influenciam seu comportamento de moagem. Dissertação de Mestrado, REDEMAT. 2004: 110p.

2 Costa RVP. Otimização da resistência à compressão de pelotas de minério de ferro para redução direta pela aplicação de projeto robusto. Dissertação de Mestrado, REDEMAT. 2008: 130p.

3 Meyer K. Pelletizing of iron ores. Springer, Velag Berlim, Heidelberg, Velag Stahleissen $\mathrm{mbH}$. Düsseldorf, Germany. 1980: 21-205.

4 Paquenet G, Stéphanie G, Éloi G. Physical test: their relevance of iron ores pellets. AISTech proceedings. 2015(1). 